\title{
Some new formulas for the products of the Apostol type polynomials
}

\author{
Yuan $\mathrm{He}^{1}$, Serkan $\mathrm{Araci}^{2^{*}}$ and HM Srivastava ${ }^{3,4}$
}

\section{"Correspondence:}

mtsrkn@hotmail.com

${ }^{2}$ Department of Economics, Faculty

of Economics, Administrative and

Social Sciences, Hasan Kalyoncu

University, Gaziantep, 27410, Turkey

Full list of author information is

available at the end of the article

\begin{abstract}
In the year 2014, Kim et al. computed a kind of new sums of the products of an arbitrary number of the classical Bernoulli and Euler polynomials by using the Euler basis for the vector space of polynomials of bounded degree. Inspired by their work, in this paper, we establish some new formulas for such a kind of sums of the products of an arbitrary number of the Apostol-Bernoulli, Euler, and Genocchi polynomials by making use of the generating function methods and summation transform techniques. The results derived here are generalizations of the corresponding known formulas involving the classical Bernoulli, Euler, and Genocchi polynomials.
\end{abstract}

MSC: Primary 11B68; secondary 05A19

Keywords: Apostol-Bernoulli polynomials; Apostol-Euler polynomials; Apostol-Genocchi polynomials; summation formulas; recurrence relations

\section{Introduction}

The classical Bernoulli polynomials $B_{n}(x)$, Euler polynomials $E_{n}(x)$, and Genocchi polynomials $G_{n}(x)$ are usually defined by the following generating functions:

$$
\begin{aligned}
& \frac{t e^{x t}}{e^{t}-1}=\sum_{n=0}^{\infty} B_{n}(x) \frac{t^{n}}{n !} \quad(|t|<2 \pi), \\
& \frac{2 e^{x t}}{e^{t}+1}=\sum_{n=0}^{\infty} E_{n}(x) \frac{t^{n}}{n !} \quad(|t|<\pi),
\end{aligned}
$$

and

$$
\frac{2 t e^{x t}}{e^{t}+1}=\sum_{n=0}^{\infty} G_{n}(x) \frac{t^{n}}{n !} \quad(|t|<\pi) .
$$

The rational numbers $B_{n}$, the integers $E_{n}$, and the rational numbers $G_{n}$ given by

$$
B_{n}=B_{n}(0), \quad E_{n}=2^{n} E_{n}\left(\frac{1}{2}\right), \quad \text { and } \quad G_{n}=G_{n}(0)
$$

are called the classical Bernoulli numbers, Euler numbers, and Genocchi numbers, respectively. These polynomials and numbers play important roles in many different areas

(c) He et al. 2016. This article is distributed under the terms of the Creative Commons Attribution 4.0 International License (http://creativecommons.org/licenses/by/4.0/), which permits unrestricted use, distribution, and reproduction in any medium, provided you give appropriate credit to the original author(s) and the source, provide a link to the Creative Commons license, and indicate if changes were made. 
of mathematics, such as number theory, combinatorics, special functions and analysis. Numerous interesting properties for them can be found in many books and papers (see, for example, [1-6]).

Some widely investigated analogs of the above classical Bernoulli, Euler and Genocchi polynomials are the Apostol-Bernoulli polynomials $\mathcal{B}_{n}(x ; \lambda)$, Apostol-Euler polynomials $\mathcal{E}_{n}(x ; \lambda)$ and Apostol-Genocchi polynomials $\mathcal{G}_{n}(x ; \lambda)$, which are usually defined by means of the following generating functions (see, e.g., [7-9]):

$$
\begin{aligned}
\frac{t e^{x t}}{\lambda e^{t}-1} & =\sum_{n=0}^{\infty} \mathcal{B}_{n}(x ; \lambda) \frac{t^{n}}{n !} \\
(|t| & <2 \pi \text { when } \lambda=1 ;|t|<|\log \lambda| \text { when } \lambda \neq 1), \\
\frac{2 e^{x t}}{\lambda e^{t}+1} & =\sum_{n=0}^{\infty} \mathcal{E}_{n}(x ; \lambda) \frac{t^{n}}{n !} \\
\quad(|t| & <\pi \text { when } \lambda=1 ;|t|<|\log (-\lambda)| \text { when } \lambda \neq 1),
\end{aligned}
$$

and

$$
\begin{aligned}
\frac{2 t e^{x t}}{\lambda e^{t}+1} & =\sum_{n=0}^{\infty} \mathcal{G}_{n}(x ; \lambda) \frac{t^{n}}{n !} \\
& (|t|<\pi \text { when } \lambda=1 ;|t|<|\log (-\lambda)| \text { when } \lambda \neq 1) .
\end{aligned}
$$

In particular, $\mathcal{B}_{n}(\lambda), \mathcal{E}_{n}(\lambda)$, and $\mathcal{G}_{n}(\lambda)$ given by

$$
\mathcal{B}_{n}(\lambda)=\mathcal{B}_{n}(0 ; \lambda), \quad \mathcal{E}_{n}(\lambda)=2^{n} \mathcal{E}_{n}\left(\frac{1}{2} ; \lambda\right), \quad \text { and } \quad \mathcal{G}_{n}(\lambda)=\mathcal{G}_{n}(0 ; \lambda)
$$

are called the Apostol-Bernoulli numbers, Apostol-Euler numbers, and Apostol-Genocchi numbers, respectively. Obviously, $\mathcal{B}_{n}(x ; \lambda), \mathcal{E}_{n}(x ; \lambda)$, and $\mathcal{G}_{n}(x ; \lambda)$ reduce, respectively, to $B_{n}(x), E_{n}(x)$, and $G_{n}(x)$ when $\lambda=1$. It is worth mentioning that the Apostol-Bernoulli polynomials were first introduced by Apostol [10] (see also Srivastava [11] for a systematic further study) in order to evaluate the value of the Hurwitz-Lerch zeta function. Since the publication of the work by Luo and Srivastava [7-9], some interesting properties for the Apostol-Bernoulli, Euler and Genocchi polynomials have been well explored by many authors (see, for example, [12-17]).

The present paper is concerned with the sums of the products of an arbitrary number of the above-mentioned polynomials and numbers. The best known such formula is Dilcher's result on the following sums of the products of an arbitrary number of the classical Bernoulli polynomials (see, for details, [18]):

$$
\begin{aligned}
& \sum_{\substack{i_{1}+\cdots+i_{k}=n \\
\left(i_{1}, \ldots, i_{k} \geq 0\right)}}\left(\begin{array}{c}
n \\
i_{1}, \ldots, i_{k}
\end{array}\right) B_{i_{1}}\left(x_{1}\right) \cdots B_{i_{k}}\left(x_{k}\right) \\
& =(-1)^{k-1} k\left(\begin{array}{l}
n \\
k
\end{array}\right) \sum_{i=0}^{k-1}(-1)^{i}\left[\sum_{j=0}^{i}\left(\begin{array}{c}
k-i-1+j \\
j
\end{array}\right) s(k, k-i+j) y^{j}\right] \frac{B_{n-i}(y)}{n-i},
\end{aligned}
$$


where $n$ and $k$ are positive integers (with $n \geqq k),\left(\begin{array}{c}n \\ i_{1}, \ldots, i_{k}\end{array}\right)$ denotes the multinomial coefficients given by

$$
\left(\begin{array}{c}
n \\
i_{1}, \ldots, i_{k}
\end{array}\right)=\frac{n !}{i_{1} ! \cdots i_{k} !}
$$

$s(n, k)$ are the Stirling numbers of the first kind and

$$
y=x_{1}+\cdots+x_{k}
$$

We refer to [19-26] for some extensions of (1.7) in different directions. In the year 2014, Kim et al. [27] considered and computed the following kind of new sums of the products of an arbitrary number of the classical Bernoulli and Euler polynomials by making use of the Euler basis for the vector space of polynomials of bounded degree:

$$
\begin{aligned}
V_{n ; r, s}(x)= & \sum_{I_{r}+J_{s}=n} \prod_{k=1}^{r} B_{i_{k}}(x) \prod_{k=1}^{s} E_{j_{k}}(x) \\
= & \frac{1}{2} \sum_{k=0}^{n-2}\left(\begin{array}{c}
n+r+s-1 \\
k
\end{array}\right) \alpha_{n, k}(r, s) E_{k}(x) \\
& +\left(\begin{array}{c}
n+r+s-1 \\
n
\end{array}\right) E_{n}(x)
\end{aligned}
$$

where $n, r$, and $s$ are positive integers,

$$
\sum_{I_{r}+J_{s}=n}
$$

denotes the sum over all non-negative integers $i_{1}, \ldots, i_{r}$ and $j_{1}, \ldots, j_{s}$ such that

$$
i_{1}+\cdots+i_{r}+j_{1}+\cdots+j_{s}=n,
$$

and $\alpha_{n, k}(r, s)$ is a rational number determined by

$$
\begin{aligned}
\alpha_{n, k}(r, s)= & \sum_{j=0}^{s} \sum_{i=\max (0, r+k-n)}^{r}\left(\begin{array}{l}
r \\
i
\end{array}\right)\left(\begin{array}{l}
s \\
j
\end{array}\right)(-1)^{j} 2^{s-j} V_{n+i-k-r ; i, j}(0) \\
& +V_{n-k ; r, s}(0) .
\end{aligned}
$$

Motivated and inspired by the work of Kim et al. [27], in this paper, we establish some new formulas for such a kind of sums of the products of an arbitrary number of the Apostol-Bernoulli, Euler and Genocchi polynomials by making use of the generating function methods and summation transform techniques. As applications, some known results for the classical Bernoulli, Euler, and Genocchi polynomials are shown to be derivable as special cases of our product formulas.

Our paper is organized as follows. In Section 2, we give several new formulas for the products of the Apostol-Bernoulli, Euler, and Genocchi polynomials. Various corollaries and consequences of these main results are also considered in Section 2 itself. Section 3 is devoted to the proofs of the main results. 


\section{Statements of the main results}

Let $r$ and $s$ be positive integers and let

$\lambda_{1}, \ldots, \lambda_{r}$ and $\mu_{1}, \ldots, \mu_{s}$

be $r+s$ parameters. For convenience, in the following, we always denote by $\lambda$ a parameter given by

$$
\lambda=\prod_{k=1}^{r} \lambda_{k} \prod_{k=1}^{s} \mu_{k},
$$

with

$$
\sum_{I_{r}+J_{s}=n}
$$

the same as in (1.10), and by $M_{a}, N_{b}$, and $T_{b}$ three sequences of polynomials given (for positive integers $a$ and $b$ ) with

$$
\begin{aligned}
& M_{a}=\prod_{k=1}^{a-1} \lambda_{k} \mathcal{B}_{i_{k}}\left(x_{k}-x_{a}+1 ; \lambda_{k}\right) \prod_{k=a+1}^{r} \mathcal{B}_{i_{k}}\left(x_{k}-x_{a} ; \lambda_{k}\right), \\
& N_{b}=\prod_{k=1}^{b-1} \mu_{k} \mathcal{E}_{j_{k}}\left(y_{k}-y_{b}+1 ; \mu_{k}\right) \prod_{k=b+1}^{s} \mathcal{E}_{j_{k}}\left(y_{k}-y_{b} ; \mu_{k}\right),
\end{aligned}
$$

and

$$
T_{b}=\prod_{k=1}^{b-1} \mu_{k} \mathcal{G}_{j_{k}}\left(y_{k}-y_{b}+1 ; \mu_{k}\right) \prod_{k=b+1}^{s} \mathcal{G}_{j_{k}}\left(y_{k}-y_{b} ; \mu_{k}\right),
$$

respectively. We also write, for subsets $R \subseteq\{1, \ldots, r\}$ and $S \subseteq\{1, \ldots, s\},|R|$ as the cardinality of $R$ and $|S|$ as the cardinality of $S, \bar{R}=\{1, \ldots, r\} \backslash R$ and $\bar{S}=\{1, \ldots, s\} \backslash S$ for positive integers $r$ and $s$. In particular, if $|R|=a$ and $|S|=b$ for positive integers $a$ and $b$, we denote $s_{1}, \ldots, s_{r-a} \in$ $\bar{R}$ and $r_{1}, \ldots, r_{s-b} \in \bar{S}$.

We now state our results as follows.

Theorem 1 Let $r$ and $s$ be positive integers. Also let $s$ be an even integer. Then, for every non-negative integer $n$,

$$
\begin{aligned}
& (n+r+s) \sum_{I_{r}+J_{s}=n} \prod_{k=1}^{r} \mathcal{B}_{i_{k}}\left(x_{k} ; \lambda_{k}\right) \prod_{k=1}^{s} \mathcal{E}_{j_{k}}\left(y_{k} ; \mu_{k}\right) \\
& =\sum_{I_{r}+J_{s}=n} \sum_{a=1}^{r}\left(\begin{array}{c}
n+r+s \\
i_{a}
\end{array}\right) \mathcal{B}_{i_{a}}\left(x_{a} ; \lambda\right) M_{a} \prod_{k=1}^{s} \mathcal{E}_{j_{k}}\left(y_{k}-x_{a} ; \mu_{k}\right) \\
& \quad+2 \sum_{I_{r}+J_{s}=n+1} \sum_{b=1}^{s}\left(\begin{array}{c}
n+r+s \\
j_{b}
\end{array}\right)(-1)^{b} \mathcal{B}_{j_{b}}\left(y_{b} ; \lambda\right) N_{b} \prod_{k=1}^{r} \lambda_{k} \mathcal{B}_{i_{k}}\left(x_{k}-y_{b}+1 ; \lambda_{k}\right) .
\end{aligned}
$$


Furthermore, if s is an odd positive integer, then, for every positive integer $n$,

$$
\begin{aligned}
\sum_{I_{r}+J_{s}=n} & \prod_{k=1}^{r} \mathcal{B}_{i_{k}}\left(x_{k} ; \lambda_{k}\right) \prod_{k=1}^{s} \mathcal{E}_{j_{k}}\left(y_{k} ; \mu_{k}\right) \\
= & -\frac{1}{2} \sum_{I_{r}+J_{s}=n-1} \sum_{a=1}^{r}\left(\begin{array}{c}
n+r+s-1 \\
i_{a}
\end{array}\right) \mathcal{E}_{i_{a}}\left(x_{a} ; \lambda\right) M_{a} \prod_{k=1}^{s} \mathcal{E}_{j_{k}}\left(y_{k}-x_{a} ; \mu_{k}\right) \\
& -\sum_{I_{r}+J_{s}=n} \sum_{b=1}^{s}\left(\begin{array}{c}
n+r+s-1 \\
j_{b}
\end{array}\right)(-1)^{b} \mathcal{E}_{j_{b}}\left(y_{b} ; \lambda\right) N_{b} \prod_{k=1}^{r} \lambda_{k} \mathcal{B}_{i_{k}}\left(x_{k}-y_{b}+1 ; \lambda_{k}\right) .
\end{aligned}
$$

We now deduce some special cases of Theorem 1. Since the Apostol-Bernoulli and Apostol-Euler polynomials satisfy the following difference equations (see, e.g., [8]):

$$
\lambda \mathcal{B}_{n}(x+1, \lambda)-\mathcal{B}_{n}(x, \lambda)=n x^{n-1} \quad(n \geqq 0)
$$

and

$$
\lambda \mathcal{E}_{n}(x+1, \lambda)+\mathcal{E}_{n}(x, \lambda)=2 x^{n} \quad(n \geqq 0),
$$

respectively, so we find from (2.7) and (2.8) that

$$
\begin{aligned}
& \prod_{k=1}^{a-1} \lambda_{k} \mathcal{B}_{i_{k}}\left(x_{k}-x_{a}+1, \lambda_{k}\right) \\
& \quad=\sum_{T \subseteq\{1, \ldots, a-1\}} \prod_{k \in T} \mathcal{B}_{i_{k}}\left(x_{k}-x_{a}, \lambda_{k}\right) \prod_{k \in \bar{T}} i_{k}\left(x_{k}-x_{a}\right)^{i_{k}-1}
\end{aligned}
$$

and

$$
\begin{aligned}
& \prod_{k=1}^{b-1}\left\{-\mu_{k} \mathcal{E}_{j_{k}}\left(y_{k}-y_{b}+1, \mu_{k}\right)\right\} \\
& \quad=\sum_{T \subseteq\{1, \ldots, b-1\}} \prod_{k \in T} \mathcal{E}_{j_{k}}\left(y_{k}-y_{b}, \mu_{k}\right) \prod_{k \in \bar{T}}\left\{-2\left(y_{k}-y_{b}\right)^{j_{k}}\right\} .
\end{aligned}
$$

Hence, by setting

$$
x_{1}=\cdots=x_{r}=x \quad \text { and } \quad y_{1}=\cdots=y_{s}=y
$$

in Theorem 1, in view of (2.9) and (2.10), we obtain the following result.

Corollary 1 Let $r$ and $s$ be positive integers. Also let $s$ be an even integer. Then, for every non-negative integer $n$,

$$
\begin{aligned}
& (n+r+s) \sum_{I_{r}+J_{s}=n} \prod_{k=1}^{r} \mathcal{B}_{i_{k}}\left(x ; \lambda_{k}\right) \prod_{k=1}^{s} \mathcal{E}_{j_{k}}\left(y ; \mu_{k}\right) \\
& =\sum_{a=1}^{r} \sum_{|R|=a} \sum_{I_{r-a}+i_{0}+J_{s}=n+1-a}\left(\begin{array}{c}
n+r+s \\
i_{0}
\end{array}\right) \mathcal{B}_{i_{0}}(x ; \lambda)
\end{aligned}
$$




$$
\begin{aligned}
& \cdot \prod_{k=1}^{r-a} \mathcal{B}_{i_{k}}\left(\lambda_{s_{k}}\right) \prod_{k=1}^{s} \mathcal{E}_{j_{k}}\left(y-x ; \mu_{k}\right) \\
& +\sum_{b=1}^{s} \sum_{|S|=b}(-2)^{b} \sum_{I_{r}+j_{0}+J_{s-b}=n+1}\left(\begin{array}{c}
n+r+s \\
j_{0}
\end{array}\right) \mathcal{B}_{j_{0}}(y ; \lambda) \\
& \cdot \prod_{k=1}^{s-b} \mathcal{E}_{j_{k}}\left(0 ; \mu_{r_{k}}\right) \prod_{k=1}^{r} \lambda_{k} \mathcal{B}_{i_{k}}\left(x-y+1 ; \lambda_{k}\right) .
\end{aligned}
$$

Moreover, if $s$ is an odd positive integer, then, for every positive integer $n$,

$$
\begin{aligned}
\sum_{I_{r}+J_{s}=n} & \prod_{k=1}^{r} \mathcal{B}_{i_{k}}\left(x ; \lambda_{k}\right) \prod_{k=1}^{s} \mathcal{E}_{j_{k}}\left(y ; \mu_{k}\right) \\
= & -\frac{1}{2} \sum_{a=1}^{r} \sum_{|R|=a} \sum_{I_{r-a}+i_{0}+J_{s}=n-a}\left(\begin{array}{c}
n+r+s-1 \\
i_{0}
\end{array}\right) \mathcal{E}_{i_{0}}(x ; \lambda) \\
& \cdot \prod_{k=1}^{r-a} \mathcal{B}_{i_{k}}\left(\lambda_{s_{k}}\right) \prod_{k=1}^{s} \mathcal{E}_{j_{k}}\left(y-x ; \mu_{k}\right) \\
& +\sum_{b=1}^{s} \sum_{|S|=b}(-2)^{b-1} \sum_{I_{r}+j_{0}+J_{s-b}=n}\left(\begin{array}{c}
n+r+s-1 \\
j_{0}
\end{array}\right) \mathcal{E}_{j_{0}}(y ; \lambda) \\
& \cdot \prod_{k=1}^{s-b} \mathcal{E}_{j_{k}}\left(0 ; \mu_{r_{k}}\right) \prod_{k=1}^{r} \lambda_{k} \mathcal{B}_{i_{k}}\left(x-y+1 ; \lambda_{k}\right) .
\end{aligned}
$$

Since the Apostol-Bernoulli polynomials satisfy the following symmetric distribution (see, e.g., [8]):

$$
\lambda \mathcal{B}_{n}(1-x ; \lambda)=(-1)^{n} \mathcal{B}_{n}\left(x ; \frac{1}{\lambda}\right) \quad(n \geqq 0),
$$

by setting

$$
\lambda_{1}=\cdots=\lambda_{r}=1 \text { and } \mu_{1}=\cdots=\mu_{s}=1
$$

in Corollary 1, we get the following formulas for the products of an arbitrary number of the classical Bernoulli polynomials and the classical Euler polynomials.

Corollary 2 Let $r$ and $s$ be positive integers. If $s$ is an even positive integer, then, for every non-negative integer $n$,

$$
\begin{aligned}
(n+r & +s) \sum_{I_{r}+J_{s}=n} \prod_{k=1}^{r} B_{i_{k}}(x) \prod_{k=1}^{s} E_{j_{k}}(y) \\
= & \sum_{a=1}^{r}\left(\begin{array}{l}
r \\
a
\end{array}\right) \sum_{I_{r-a}+i_{0}+J_{s}=n+1-a}\left(\begin{array}{c}
n+r+s \\
i_{0}
\end{array}\right) B_{i_{0}}(x) \prod_{k=1}^{r-a} B_{i_{k}} \prod_{k=1}^{s} E_{j_{k}}(y-x) \\
& +\sum_{b=1}^{s}\left(\begin{array}{l}
s \\
b
\end{array}\right)(-2)^{b} \sum_{I_{r}+j_{0}+J_{s-b}=n+1}\left(\begin{array}{c}
n+r+s \\
j_{0}
\end{array}\right)(-1)^{I_{r}} B_{j_{0}}(y) \\
& \cdot \prod_{k=1}^{s-b} E_{j_{k}}(0) \prod_{k=1}^{r} B_{i_{k}}(y-x) .
\end{aligned}
$$


Furthermore, if s is an odd positive integer, then, for every positive integer $n$,

$$
\begin{aligned}
\sum_{I_{r}+J_{s}=n} & \prod_{k=1}^{r} B_{i_{k}}(x) \prod_{k=1}^{s} E_{j_{k}}(y) \\
= & -\frac{1}{2} \sum_{a=1}^{r}\left(\begin{array}{l}
r \\
a
\end{array}\right) \sum_{I_{r-a}+i_{0}+J_{s}=n-a}\left(\begin{array}{c}
n+r+s-1 \\
i_{0}
\end{array}\right) E_{i_{0}}(x) \\
& \cdot \prod_{k=1}^{r-a} B_{i_{k}} \prod_{k=1}^{s} E_{j_{k}}(y-x) \\
& +\sum_{b=1}^{s}\left(\begin{array}{l}
s \\
b
\end{array}\right)(-2)^{b-1} \sum_{I_{r}+j_{0}+J_{s-b}=n}\left(\begin{array}{c}
n+r+s-1 \\
j_{0}
\end{array}\right)(-1)^{I_{r}} E_{j_{0}}(y) \\
& \cdot \prod_{k=1}^{s-b} E_{j_{k}}(0) \prod_{k=1}^{r} B_{i_{k}}(y-x) .
\end{aligned}
$$

In the special case when $x=y$, Corollary 2 yields the corresponding new expressions for the above-mentioned sums of the products of an arbitrary number of the classical Bernoulli polynomials and the classical Euler polynomials considered by Kim et al. [27]. If we take $r=s=1$ in Corollary 1, in light of (2.7), we obtain the following result.

Corollary 3 Let $n$ be a positive integer. Then

$$
\begin{aligned}
\sum_{k=0}^{n} \mathcal{B}_{k}(x ; \lambda) \mathcal{E}_{n-k}(y ; \mu) \\
=-\frac{1}{2} \sum_{k=0}^{n-1}\left(\begin{array}{l}
n+1 \\
k+2
\end{array}\right) \mathcal{E}_{n-1-k}(x ; \lambda \mu) \mathcal{E}_{k}(y-x ; \mu) \\
\quad+\sum_{k=0}^{n}\left(\begin{array}{l}
n+1 \\
k+1
\end{array}\right) \mathcal{E}_{n-k}(y ; \lambda \mu)\left\{\mathcal{B}_{k}(x-y ; \lambda)+k(x-y)^{k-1}\right\} .
\end{aligned}
$$

In particular, since (see, e.g., [28])

$$
E_{n}(0)=2\left(1-2^{n+1}\right) \frac{B_{n+1}}{n+1} \quad(n \geqq 0),
$$

by setting

$$
x=y \quad \text { and } \quad \lambda=\mu=1
$$

in Corollary 3, we find for every positive integer $n \geqq 2$ that

$$
\begin{aligned}
& \sum_{k=0}^{n} B_{k}(x) E_{n-k}(x)-\sum_{k=2}^{n}\left(\begin{array}{c}
n+1 \\
k+1
\end{array}\right)\left(2^{k}+k-1\right) \frac{B_{k}}{k} E_{n-k}(x) \\
& \quad=(n+1) E_{n}(x),
\end{aligned}
$$

which was derived by Pan and Sun [29] by using the finite difference calculus and differentiation. 
Theorem 2 Let $r$ and $s$ be positive integers. Then, for every non-negative integer $n$,

$$
\begin{aligned}
& (n+r+s) \sum_{I_{r}+J_{s}=n} \prod_{k=1}^{r} \mathcal{B}_{i_{k}}\left(x_{k} ; \lambda_{k}\right) \prod_{k=1}^{s} \mathcal{G}_{j_{k}}\left(y_{k} ; \mu_{k}\right) \\
& =\sum_{I_{r}+J_{s}=n} \sum_{a=1}^{r}\left(\begin{array}{c}
n+r+s \\
i_{a}
\end{array}\right) \mathcal{P}_{i_{a}}\left(x_{a} ; \lambda\right) M_{a} \prod_{k=1}^{s} \mathcal{G}_{j_{k}}\left(y_{k}-x_{a} ; \mu_{k}\right) \\
& \quad+2 \sum_{I_{r}+J_{s}=n} \sum_{b=1}^{s}\left(\begin{array}{c}
n+r+s \\
j_{b}
\end{array}\right)(-1)^{b} \mathcal{P}_{j_{b}}\left(y_{b} ; \lambda\right) T_{b} \\
& \quad \cdot \prod_{k=1}^{r}\left\{\lambda_{k} \mathcal{B}_{i_{k}}\left(x_{k}-y_{b}+1 ; \lambda_{k}\right)\right\}
\end{aligned}
$$

where $\mathcal{P}_{n}(x ; \lambda)$ is given by

$$
\mathcal{P}_{n}(x ; \lambda)= \begin{cases}\mathcal{B}_{n}(x ; \lambda) & (2 \mid s), \\ -\frac{1}{2} \mathcal{G}_{n}(x ; \lambda) & (2 \nmid s) .\end{cases}
$$

We now deduce some special cases of Theorem 2. Since the Apostol-Genocchi polynomials satisfy the following difference equation (see, e.g., [7]):

$$
\lambda \mathcal{G}_{n}(x+1, \lambda)+\mathcal{G}_{n}(x, \lambda)=2 n x^{n-1} \quad(n \geqq 0),
$$

by applying (2.19), we have

$$
\begin{aligned}
& \prod_{k=1}^{b-1}\left\{-\mu_{k} \mathcal{G}_{j_{k}}\left(y_{k}-y_{b}+1, \mu_{k}\right)\right\} \\
& \quad=\sum_{T \subseteq\{1, \ldots, b-1\}} \prod_{k \in T} \mathcal{G}_{j_{k}}\left(y_{k}-y_{b}, \mu_{k}\right) \prod_{k \in T}\left\{-2 j_{k}\left(y_{k}-y_{b}\right)^{j_{k}-1}\right\} .
\end{aligned}
$$

Hence, by setting

$$
x_{1}=\cdots=x_{r}=x \quad \text { and } \quad y_{1}=\cdots=y_{s}=y
$$

in Theorem 2, and in view of (2.9) and (2.20), we obtain the following result.

Corollary 4 Let $r$ and $s$ be positive integers. Then, for every non-negative integer $n$,

$$
\begin{aligned}
(n+r+s) & \sum_{I_{r}+J_{s}=n} \prod_{k=1}^{r} \mathcal{B}_{i_{k}}\left(x ; \lambda_{k}\right) \prod_{k=1}^{s} \mathcal{G}_{j_{k}}\left(y ; \mu_{k}\right) \\
= & \sum_{a=1}^{r} \sum_{|R|=a} \sum_{I_{r-a}+i_{0}+J_{s}=n+1-a}\left(\begin{array}{c}
n+r+s \\
i_{0}
\end{array}\right) \mathcal{P}_{i_{0}}(x ; \lambda) \\
& \cdot \prod_{k=1}^{r-a} \mathcal{B}_{i_{k}}\left(\lambda_{s_{k}}\right) \prod_{k=1}^{s} \mathcal{G}_{j_{k}}\left(y-x ; \mu_{k}\right)
\end{aligned}
$$




$$
\begin{aligned}
& +\sum_{b=1}^{s} \sum_{|S|=b}(-2)^{b} \sum_{I_{r}+j_{0}+J_{s-b}=n+1-b}\left(\begin{array}{c}
n+r+s \\
j_{0}
\end{array}\right) \mathcal{P}_{j_{0}}(y ; \lambda) \\
& \cdot \prod_{k=1}^{s-b} \mathcal{G}_{j_{k}}\left(\mu_{r_{k}}\right) \prod_{k=1}^{r}\left\{\lambda_{k} \mathcal{B}_{i_{k}}\left(x-y+1 ; \lambda_{k}\right)\right\} .
\end{aligned}
$$

Upon setting

$$
\lambda_{1}=\cdots=\lambda_{r}=1 \quad \text { and } \quad \mu_{1}=\cdots=\mu_{s}=1
$$

in Corollary 4, if we make use of (2.13), we obtain the following formula for the products of an arbitrary number of the classical Bernoulli and Genocchi polynomials.

Corollary 5 Let $r$ and s be positive integers. Then, for every non-negative integer $n$,

$$
\begin{aligned}
(n+r & +s) \sum_{I_{r}+J_{s}=n} \prod_{k=1}^{r} B_{i_{k}}(x) \prod_{k=1}^{s} G_{j_{k}}(y) \\
= & \sum_{a=1}^{r}\left(\begin{array}{l}
r \\
a
\end{array}\right) \sum_{I_{r-a}+i_{0}+J_{s}=n+1-a}\left(\begin{array}{c}
n+r+s \\
i_{0}
\end{array}\right) P_{i_{0}}(x) \prod_{k=1}^{r-a} B_{i_{k}} \prod_{k=1}^{s} G_{j_{k}}(y-x) \\
& +\sum_{b=1}^{s}\left(\begin{array}{l}
s \\
b
\end{array}\right)(-2)^{b} \sum_{I_{r}+j_{0}+J_{s-b}=n+1-b}\left(\begin{array}{c}
n+r+s \\
j_{0}
\end{array}\right)(-1)^{I_{r}} P_{j_{0}}(y) \\
& \cdot \prod_{k=1}^{s-b} G_{j_{k}} \prod_{k=1}^{r} B_{i_{k}}(y-x)
\end{aligned}
$$

where $P_{n}(x)$ is given by

$$
P_{n}(x)= \begin{cases}B_{n}(x) & (2 \mid s), \\ -\frac{1}{2} G_{n}(x) & (2 \nmid s) .\end{cases}
$$

If we take $r=s=1$ in Corollary 4, in light of (2.7), we get the following result.

Corollary 6 Let $n$ be a non-negative integer. Then

$$
\begin{aligned}
\sum_{k=0}^{n} \mathcal{B}_{k}(x ; \lambda) \mathcal{G}_{n-k}(y ; \mu) \\
=-\frac{1}{2} \sum_{k=1}^{n} \frac{1}{k}\left(\begin{array}{c}
n+1 \\
k-1
\end{array}\right)\left[\mathcal{G}_{k}(x ; \lambda \mu) \mathcal{G}_{n-k}(y-x ; \mu)\right] \\
\quad+\sum_{k=1}^{n} \frac{1}{k}\left(\begin{array}{c}
n+1 \\
k-1
\end{array}\right)\left(\mathcal{G}_{k}(y ; \lambda \mu)\left[\mathcal{B}_{n-k}(x-y ; \lambda)+(n-k)(x-y)^{n-k-1}\right]\right) .
\end{aligned}
$$

Since the classical Genocchi polynomials can be expressed in terms of the classical Bernoulli polynomials as follows:

$$
G_{n}(x)=2 B_{n}(x)-2^{n+1} B_{n}\left(\frac{x}{2}\right) \quad(n \geqq 0),
$$


by setting $\lambda=\mu=1$ and $x=y$ in Corollary 6 , and in light of the fact that (see, e.g., [7, 28])

$$
B_{0}=1, \quad B_{1}=-\frac{1}{2} \quad \text { and } \quad G_{0}(x)=0,
$$

we find for every positive integer $n \geqq 3$ that

$$
\begin{aligned}
& \sum_{k=1}^{n-1} B_{k}(x) G_{n-k}(x)-\sum_{k=1}^{n-2} \frac{1}{k}\left(\begin{array}{c}
n+1 \\
k-1
\end{array}\right)\left[2^{n-k} G_{k}(x) B_{n-k}\right] \\
& \quad=\frac{1}{2}(n-1) G_{n}(x),
\end{aligned}
$$

which was derived by Agoh [30] by applying some short and intelligible ideas. For some convolution formulas similar to (2.17) and (2.25), the interested reader may be referred to [31-36].

\section{Proofs of Theorems 1 and 2}

In our proofs of Theorems 1 and 2, we need the following auxiliary result described in $[37,38]$.

Lemma 1 Let $n$ be a positive integer with $n \geqq 2$ and let $\Omega_{n}$ be the $n$-dimensional space (or the standard simplex in $\mathbb{R}^{n}$ ) defined by

$$
\Omega_{n}:=\left\{\left(t_{1}, \ldots, t_{n}\right): t_{k} \geqq 0(k=1, \ldots, n) \text { and } t_{1}+\cdots+t_{n} \leqq 1\right\} .
$$

Then the multivariable Beta function $B\left(\alpha_{1}, \ldots, \alpha_{n}\right)$ is given by the following Dirichlet integral:

$$
\begin{aligned}
B\left(\alpha_{1}, \ldots, \alpha_{n}\right)= & \frac{\Gamma\left(\alpha_{1}\right) \cdots \Gamma\left(\alpha_{n}\right)}{\Gamma\left(\alpha_{1}+\cdots+\alpha_{n}\right)} \\
= & \int \cdots \int_{\Omega_{n-1}} t_{1}^{\alpha_{1}-1} \cdots t_{n-1}^{\alpha_{n-1}-1} \\
& \cdot\left(1-t_{1}-\cdots-t_{n-1}\right)^{\alpha_{n}-1} d t_{1} \cdots d t_{n-1} \\
& \left(\min \left\{\Re\left(\alpha_{1}\right), \ldots, \Re\left(\alpha_{n}\right)\right\}>0\right) .
\end{aligned}
$$

Proof of Theorem 1 We first recall the following elementary and beautiful idea:

$$
\begin{aligned}
& \left(1+x_{1}\right)\left(1+x_{2}\right)\left(1+x_{3}\right) \cdots \\
& \quad=\left(1+x_{1}\right)+x_{2}\left(1+x_{1}\right)+x_{3}\left(1+x_{1}\right)\left(1+x_{2}\right)+\cdots,
\end{aligned}
$$

which was used by Euler to give the proof of his famous pentagonal number theorem (see, e.g., $[39,40])$. Obviously, the finite form of (3.2) can be expressed as follows:

$$
\begin{aligned}
& \left(1+x_{1}\right) \cdots\left(1+x_{n}\right) \\
& \quad=\left(1+x_{1}\right)+x_{2}\left(1+x_{1}\right)+\cdots+x_{n}\left(1+x_{1}\right) \cdots\left(1+x_{n-1}\right) .
\end{aligned}
$$


For $1 \leqq k \leqq n$, if we write $x_{k}-1$ for $x_{k}$ in (3.3), we get

$$
x_{1} \cdots x_{n}-1=\sum_{k=1}^{n}\left(x_{k}-1\right) x_{1} \cdots x_{k-1} \text {, }
$$

where the product $x_{1} \cdots x_{k-1}$ is assumed to be equal to 1 when $k=1$. Let $\varepsilon_{k}$ be a piecewise function of $k$ given by

$$
\varepsilon_{k}= \begin{cases}\lambda_{k} & (1 \leqq k \leqq r), \\ -\mu_{k-r} & (r+1 \leqq k \leqq r+s) .\end{cases}
$$

By replacing $n$ by $r+s$ and taking $x_{k}=\varepsilon_{k} e^{t_{k}}$ in (3.4), we find that

$$
\begin{aligned}
& (-1)^{s} \lambda e^{t_{1}+\cdots+t_{r+s}}-1 \\
& =\sum_{k=1}^{r}\left(\varepsilon_{k} e^{t_{k}}-1\right) \prod_{i=1}^{k-1} \varepsilon_{i} e^{t_{i}}+\sum_{k=1}^{s}\left(\varepsilon_{r+k} e^{t_{r+k}}-1\right) \prod_{i=1}^{r+k-1} \varepsilon_{i} e^{t_{i}},
\end{aligned}
$$

which, together with (3.5), yields

$$
\begin{aligned}
& (-1)^{s} \lambda e^{t_{1}+\cdots+t_{r+s}}-1 \\
& =\sum_{a=1}^{r}\left(\lambda_{a} e^{t_{a}}-1\right) \prod_{i=1}^{a-1} \lambda_{i} e^{t_{i}}+\sum_{b=1}^{s}(-1)^{b}\left(\mu_{b} e^{t_{r+b}}+1\right) \prod_{i=1}^{b-1} \mu_{i} e^{t_{r+i}} \prod_{i=1}^{r} \lambda_{i} e^{t_{i}} .
\end{aligned}
$$

It follows from (3.7) that

$$
\begin{aligned}
\prod_{k=1}^{r} \frac{t_{k} e^{x_{k} t_{k}}}{\lambda_{k} e^{t_{k}}-1} \prod_{k=1}^{s} \frac{2 e^{y_{k} t_{r+k}}}{\mu_{k} e^{t_{r+k}}+1} \\
=\frac{1}{(-1)^{s} \lambda e^{t_{1}+\cdots+t_{r+s}}-1}\left(\sum_{a=1}^{r}\left(\lambda_{a} e^{t_{a}}-1\right) \prod_{i=1}^{a-1} \lambda_{i} e^{t_{i}}\right. \\
\cdot \prod_{k=1}^{r} \frac{t_{k} e^{x_{k} t_{k}}}{\lambda_{k} e^{t_{k}}-1} \prod_{k=1}^{s} \frac{2 e^{y_{k} t_{r+k}}}{\mu_{k} e^{t_{r+k}+1}} \\
+\sum_{b=1}^{s}(-1)^{b}\left(\mu_{b} e^{t_{r+b}}+1\right) \prod_{i=1}^{b-1} \mu_{i} e^{t_{r+i}} \\
\left.\cdot \prod_{k=1}^{r} \lambda_{k} \frac{t_{k} e^{\left(x_{k}+1\right) t_{k}}}{\lambda_{k} e^{t_{k}}-1} \prod_{k=1}^{s} \frac{2 e^{y_{k} t_{r+k}}}{\mu_{k} e^{t_{r+k}+1}}\right) .
\end{aligned}
$$

We now observe that

$$
\begin{aligned}
& \left(\lambda_{a} e^{t_{a}}-1\right) \prod_{i=1}^{a-1} \lambda_{i} e^{t_{i}} \prod_{k=1}^{r} \frac{t_{k} e^{x_{k} t_{k}}}{\lambda_{k} e^{t_{k}}-1} \\
& =t_{a} e^{x_{a}\left(t_{1}+\cdots+t_{r}\right)} \prod_{k=1}^{a-1} \lambda_{k} \frac{t_{k} e^{\left(x_{k}-x_{a}+1\right) t_{k}}}{\lambda_{k} e^{t_{k}}-1} \prod_{k=a+1}^{r} \frac{t_{k} e^{\left(x_{k}-x_{a}\right) t_{k}}}{\lambda_{k} e^{t_{k}}-1}
\end{aligned}
$$


and

$$
\begin{aligned}
& \left(\mu_{b} e^{t_{r+b}}+1\right) \prod_{i=1}^{b-1} \mu_{i} e^{t_{r+i}} \prod_{k=1}^{s} \frac{2 e^{y_{k} t_{r+k}}}{\mu_{k} e^{t_{r+k}+1}} \\
& =2 e^{y_{b}\left(t_{r+1}+\cdots+t_{r+s}\right)} \prod_{k=1}^{b-1} \mu_{k} \frac{2 e^{\left(y_{k}-y_{b}+1\right) t_{r+k}}}{\mu_{k} e^{t_{r+k}+1}} \prod_{k=b+1}^{s} \frac{2 e^{\left(y_{k}-y_{b}\right) t_{r+k}}}{\mu_{k} e^{t_{r+k}+1}} .
\end{aligned}
$$

Thus, by applying (3.9) and (3.10) to (3.8), we obtain

$$
\begin{aligned}
\prod_{k=1}^{r} \frac{t_{k} e^{x_{k} t_{k}}}{\lambda_{k} e^{t_{k}}-1} \prod_{k=1}^{s} \frac{2 e^{y_{k} t_{r+k}}}{\mu_{k} e^{t_{r+k}+1}} \\
=\sum_{a=1}^{r} \frac{t_{a} e^{x_{a}\left(t_{1}+\cdots+t_{r+s}\right)}}{(-1)^{s} \lambda e^{t_{1}+\cdots+t_{r+s}}-1} \prod_{k=1}^{a-1} \lambda_{k} \frac{t_{k} e^{\left(x_{k}-x_{a}+1\right) t_{k}}}{\lambda_{k} e^{t_{k}}-1} \\
\quad \cdot \prod_{k=a+1}^{r} \frac{t_{k} e^{\left(x_{k}-x_{a}\right) t_{k}}}{\lambda_{k} e^{t_{k}}-1} \prod_{k=1}^{s} \frac{2 e^{\left(y_{k}-x_{a}\right) t_{r+k}}}{\mu_{k} e^{t_{r+k}+1}} \\
\quad+\sum_{b=1}^{s}(-1)^{b} \frac{2 e^{y_{b}\left(t_{1}+\cdots+t_{r+s}\right)}}{(-1)^{s} \lambda e^{t_{1}+\cdots+t_{r+s}}-1} \prod_{k=1}^{b-1} \mu_{k} \frac{2 e^{\left(y_{k}-y_{b}+1\right) t_{r+k}}}{\mu_{k} e^{t_{r+k}+1}} \\
\quad \cdot \prod_{k=b+1}^{s} \frac{2 e^{\left(y_{k}-y_{b}\right) t_{r+k}}}{\mu_{k} e^{t_{r+k}+1}} \prod_{k=1}^{r} \lambda_{k} \frac{t_{k} e^{\left(x_{k}-y_{b}+1\right) t_{k}}}{\lambda_{k} e^{t_{k}-1}} .
\end{aligned}
$$

For convenience, let

$$
\left[\frac{t^{n}}{n !}\right] f(t)
$$

denote the coefficient of $\frac{t^{n}}{n !}$ in the power-series expansion of $f(t)$. For $1 \leqq k \leqq r+s$, if we substitute $u_{k} t$ for $t_{k}$ with

$$
u_{1}+\cdots+u_{r+s}=1
$$

into both sides of (3.11), we find that

$$
\begin{aligned}
{\left[\frac{t^{n}}{n !}\right] } & \left(\prod_{k=1}^{r} \frac{u_{k} t e^{x_{k} u_{k} t}}{\lambda_{k} e^{u_{k} t}-1} \prod_{k=1}^{s} \frac{2 e^{y_{k} u_{r+k} t}}{\mu_{k} e^{u_{r+k} t}+1}\right) \\
= & {\left[\frac{t^{n}}{n !}\right]\left(\sum_{a=1}^{r} \frac{u_{a} t e^{x_{a} t}}{(-1)^{s} \lambda e^{t}-1} \prod_{k=1}^{a-1} \lambda_{k} \frac{u_{k} t e^{\left(x_{k}-x_{a}+1\right) u_{k} t}}{\lambda_{k} e^{u_{k} t}-1}\right.} \\
& \left.\cdot \prod_{k=a+1}^{r} \frac{u_{k} t e^{\left(x_{k}-x_{a}\right) u_{k} t}}{\lambda_{k} e^{u_{k} t}-1} \prod_{k=1}^{s} \frac{2 e^{\left(y_{k}-x_{a}\right) u_{r+k} t}}{\mu_{k} e^{u_{r+k} t}+1}\right) \\
+ & {\left[\frac{t^{n}}{n !}\right]\left(\sum_{b=1}^{s}(-1)^{b} \frac{2 e^{y_{b} t}}{(-1)^{s} \lambda e^{t}-1} \prod_{k=1}^{b-1} \mu_{k} \frac{2 e^{\left(y_{k}-y_{b}+1\right) u_{r+k} t}}{\mu_{k} e^{u_{r+k} t}+1}\right.} \\
& \left.\cdot \prod_{k=b+1}^{s} \frac{2 e^{\left(y_{k}-y_{b}\right) u_{r+k} t}}{\mu_{k} e^{u_{r+k} t}+1} \prod_{k=1}^{r} \lambda_{k} \frac{u_{k} t e^{\left(x_{k}-y_{b}+1\right) u_{k} t}}{\lambda_{k} e^{u_{k} t}-1}\right)=: \mathrm{M}_{1}+\mathrm{M}_{2} .
\end{aligned}
$$


The left-hand side of (3.12) can easily be rewritten as follows:

$$
\begin{aligned}
& {\left[\frac{t^{n}}{n !}\right]\left(\prod_{k=1}^{r} \frac{u_{k} t e^{x_{k} u_{k} t}}{\lambda_{k} e^{u_{k} t}-1} \prod_{k=1}^{s} \frac{2 e^{y_{k} u_{r+k} t}}{\mu_{k} e^{u_{r+k} t}+1}\right)} \\
& =n ! \cdot \sum_{I_{r}+J_{s}=n} \prod_{k=1}^{r} \mathcal{B}_{i_{k}}\left(x_{k} ; \lambda_{k}\right) \frac{u_{k}^{i_{k}}}{i_{k} !} \prod_{k=1}^{s} \mathcal{E}_{j_{k}}\left(y_{k} ; \mu_{k}\right) \frac{u_{r+k}^{j_{k}}}{j_{k} !} .
\end{aligned}
$$

Moreover, $\mathrm{M}_{1}$ and $\mathrm{M}_{2}$ on the right-hand side of (3.12) can be rewritten as follows:

$$
\begin{aligned}
\mathrm{M}_{1}= & n ! \cdot \sum_{I_{r}+J_{s}=n+\epsilon} \sum_{a=1}^{r} \mathcal{F}_{i_{a}}\left(x_{a} ; \lambda\right) \frac{u_{a}}{i_{a} !} \prod_{k=1}^{a-1} \lambda_{k} \mathcal{B}_{i_{k}}\left(x_{k}-x_{a}+1 ; \lambda_{k}\right) \frac{u_{k}^{i_{k}}}{i_{k} !} \\
& \cdot \prod_{k=a+1}^{r} \mathcal{B}_{i_{k}}\left(x_{k}-x_{a} ; \lambda_{k}\right) \frac{u_{k}^{i_{k}}}{i_{k} !} \prod_{k=1}^{s} \mathcal{E}_{j_{k}}\left(y_{k}-x_{a} ; \mu_{k}\right) \frac{u_{r+k}^{j_{k}}}{j_{k} !}
\end{aligned}
$$

and

$$
\begin{aligned}
\mathrm{M}_{2}= & \cdot n ! \cdot \sum_{I_{r}+J_{s}=n+1+\epsilon} \sum_{b=1}^{s}(-1)^{b} \mathcal{F}_{j_{b}}\left(y_{b} ; \lambda\right) \frac{u_{r+b}^{0}}{j_{b} !} \\
& \cdot \prod_{k=1}^{b-1} \mu_{k} \mathcal{E}_{j_{k}}\left(y_{k}-y_{b}+1 ; \mu_{k}\right) \frac{u_{r+k}^{j_{k}}}{j_{k} !} \prod_{k=b+1}^{s} \mathcal{E}_{j_{k}}\left(y_{k}-y_{b} ; \mu_{k}\right) \frac{u_{r+k}^{j_{k}}}{j_{k} !} \\
& \cdot \prod_{k=1}^{r} \lambda_{k} \mathcal{B}_{i_{k}}\left(x_{k}-y_{b}+1 ; \lambda_{k}\right) \frac{u_{k}^{i_{k}}}{i_{k} !}
\end{aligned}
$$

where

$$
\epsilon= \begin{cases}0 & (s=2,4,6, \ldots) \\ -1 & (s=1,3,5, \ldots)\end{cases}
$$

and $\mathcal{F}_{n}(x ; \lambda)$ is determined by

$$
\mathcal{F}_{n}(x ; \lambda)= \begin{cases}\mathcal{B}_{n}(x ; \lambda) & (2 \mid s), \\ -\frac{1}{2} \mathcal{E}_{n}(x ; \lambda) & (2 \nmid s) .\end{cases}
$$

It follows from (3.12) to (3.15) that

$$
\begin{aligned}
\sum_{I_{r}+J_{s}=n} & \prod_{k=1}^{r} \mathcal{B}_{i_{k}}\left(x_{k} ; \lambda_{k}\right) \frac{u_{k}^{i_{k}}}{i_{k} !} \prod_{k=1}^{s} \mathcal{E}_{j_{k}}\left(y_{k} ; \mu_{k}\right) \frac{u_{r+k}^{j_{k}}}{j_{k} !} \\
= & \sum_{I_{r}+J_{s}=n+\epsilon} \sum_{a=1}^{r} \mathcal{F}_{i_{a}}\left(x_{a} ; \lambda\right) \frac{u_{a}}{i_{a} !} \prod_{k=1}^{a-1} \lambda_{k} \mathcal{B}_{i_{k}}\left(x_{k}-x_{a}+1 ; \lambda_{k}\right) \frac{u_{k}^{i_{k}}}{i_{k} !} \\
& \cdot \prod_{k=a+1}^{r} \mathcal{B}_{i_{k}}\left(x_{k}-x_{a} ; \lambda_{k}\right) \frac{u_{k}^{i_{k}}}{i_{k} !} \prod_{k=1}^{s} \mathcal{E}_{j_{k}}\left(y_{k}-x_{a} ; \mu_{k}\right) \frac{u_{r+k}^{j_{k}}}{j_{k} !}
\end{aligned}
$$




$$
\begin{aligned}
& +2 \sum_{I_{r}+j_{s}=n+1+\epsilon} \sum_{b=1}^{s}(-1)^{b} \mathcal{F}_{j_{b}}\left(y_{b} ; \lambda\right) \frac{u_{r+b}^{0}}{j_{b} !} \\
& \cdot \prod_{k=1}^{b-1} \mu_{k} \mathcal{E}_{j_{k}}\left(y_{k}-y_{b}+1 ; \mu_{k}\right) \frac{u_{r+k}^{j_{k}}}{j_{k} !} \prod_{k=b+1}^{s} \mathcal{E}_{j_{k}}\left(y_{k}-y_{b} ; \mu_{k}\right) \frac{u_{r+k}^{j_{k}}}{j_{k} !} \\
& \cdot \prod_{k=1}^{r} \lambda_{k} \mathcal{B}_{i_{k}}\left(x_{k}-y_{b}+1 ; \lambda_{k}\right) \frac{u_{k}^{i_{k}}}{i_{k} !}
\end{aligned}
$$

We note that, for complex numbers $\alpha_{1}, \ldots, \alpha_{r+s}$ with

$$
\min \left\{\Re\left(\alpha_{1}\right), \ldots, \Re\left(\alpha_{r+s}\right)\right\}>-1,
$$

if we use Lemma 1, we find for

$$
u_{1}+\cdots+u_{r+s}=1
$$

that

$$
\begin{aligned}
& \int \cdots \int_{\Omega_{r+s-1}} u_{1}^{\alpha_{1}} \cdots u_{r+s}^{\alpha_{r+s}} d u_{1} \cdots d u_{r+s-1} \\
& =\frac{\Gamma\left(\alpha_{1}+1\right) \cdots \Gamma\left(\alpha_{r+s}+1\right)}{\Gamma\left(\alpha_{1}+\cdots+\alpha_{r+s}+r+s\right)} .
\end{aligned}
$$

Consequently, by the following operation:

$$
\int \cdots \int_{\Omega_{r+s-1}}(\cdots) d u_{1} \cdots d u_{r+s-1}
$$

applied to both sides of (3.17), and with the help of (3.18), we get

$$
\begin{aligned}
& \frac{1}{(n+r+s-1) !} \sum_{I_{r}+J_{s}=n} \prod_{k=1}^{r} \mathcal{B}_{i_{k}}\left(x_{k} ; \lambda_{k}\right) \prod_{k=1}^{s} \mathcal{E}_{j_{k}}\left(y_{k} ; \mu_{k}\right) \\
& =\sum_{I_{r}+J_{s}=n+\epsilon} \sum_{a=1}^{r} \frac{\mathcal{F}_{i_{a}}\left(x_{a} ; \lambda\right)}{i_{a} ! \cdot\left(n+\epsilon-i_{a}+r+s\right) !} \prod_{k=1}^{a-1} \lambda_{k} \mathcal{B}_{i_{k}}\left(x_{k}-x_{a}+1 ; \lambda_{k}\right) \\
& \cdot \prod_{k=a+1}^{r} \mathcal{B}_{i_{k}}\left(x_{k}-x_{a} ; \lambda_{k}\right) \prod_{k=1}^{s} \mathcal{E}_{j_{k}}\left(y_{k}-x_{a} ; \mu_{k}\right) \\
& \quad+2 \sum_{I_{r}+J_{s}=n+1+\epsilon} \sum_{b=1}^{s}(-1)^{b} \frac{\mathcal{F}_{j_{b}}\left(y_{b} ; \lambda\right)}{j_{b} ! \cdot\left(n+\epsilon-j_{b}+r+s\right) !} \\
& \quad \cdot \prod_{k=1}^{b-1} \mu_{k} \mathcal{E}_{j_{k}}\left(y_{k}-y_{b}+1 ; \mu_{k}\right) \\
& \quad \cdot \prod_{k=b+1}^{s} \mathcal{E}_{j_{k}}\left(y_{k}-y_{b} ; \mu_{k}\right) \prod_{k=1}^{r} \lambda_{k} \mathcal{B}_{i_{k}}\left(x_{k}-y_{b}+1 ; \lambda_{k}\right)
\end{aligned}
$$

which, together with (3.16), yields the desired results (2.5) and (2.6). This completes the proof of Theorem 1. 
Proof of Theorem 2 Let $u_{1}, \ldots, u_{r+s}$ be $r+s$ variables with

$$
u_{1}+\cdots+u_{r+s}=1
$$

For $1 \leqq k \leqq s$, if we substitute $2 u_{r+k} t e^{y_{k} u_{r+k} t}$ for $2 e^{y_{k} u_{r+k} t}$ in both sides of (3.12), we find that

$$
\begin{aligned}
{\left[\frac{t^{n}}{n !}\right] } & \left(\prod_{k=1}^{r} \frac{u_{k} t e^{x_{k} u_{k} t}}{\lambda_{k} e^{u_{k} t}-1} \prod_{k=1}^{s} \frac{2 u_{r+k} t e^{y_{k} u_{r+k} t}}{\mu_{k} e^{u_{r+k} t}+1}\right) \\
= & {\left[\frac{t^{n}}{n !}\right]\left(\sum_{a=1}^{r} \frac{u_{a} t e^{x_{a} t}}{(-1)^{s} \lambda e^{t}-1} \prod_{k=1}^{a-1} \lambda_{k} \frac{u_{k} t e^{\left(x_{k}-x_{a}+1\right) u_{k} t}}{\lambda_{k} e^{u_{k} t}-1}\right.} \\
& \left.\cdot \prod_{k=a+1}^{r} \frac{u_{k} t e^{\left(x_{k}-x_{a}\right) u_{k} t}}{\lambda_{k} e^{u_{k} t}-1} \prod_{k=1}^{s} \frac{2 u_{r+k} t e^{\left(y_{k}-x_{a}\right) u_{r+k} t}}{\mu_{k} e^{u_{r+k} t}+1}\right) \\
& +\left[\frac{t^{n}}{n !}\right]\left(\sum_{b=1}^{s}(-1)^{b} \frac{2 u_{r+b} t e^{y_{b} t}}{(-1)^{s} \lambda e^{t}-1} \prod_{k=1}^{b-1} \mu_{k} \frac{2 u_{r+k} t e^{\left(y_{k}-y_{b}+1\right) u_{r+k} t}}{\mu_{k} e^{u_{r+k} t}+1}\right. \\
& \left.\cdot \prod_{k=b+1}^{s} \frac{2 u_{r+k} t e^{\left(y_{k}-y_{b}\right) u_{r+k} t}}{\mu_{k} e^{u_{r+k} t}+1} \prod_{k=1}^{r} \lambda_{k} \frac{u_{k} t e^{\left(x_{k}-y_{b}+1\right) u_{k} t}}{\lambda_{k} e^{u_{k} t}-1}\right)=\mathrm{N}_{1}+\mathrm{N}_{2},
\end{aligned}
$$

say. It is trivial to obtain

$$
\begin{aligned}
& {\left[\frac{t^{n}}{n !}\right]\left(\prod_{k=1}^{r} \frac{u_{k} t e^{x_{k} u_{k} t}}{\lambda_{k} e^{u_{k} t}-1} \prod_{k=1}^{s} \frac{2 u_{r+k} t e^{y_{k} u_{r+k} t}}{\mu_{k} e^{u_{r+k} t}+1}\right)} \\
& =n ! \cdot \sum_{I_{r}+J_{s}=n} \prod_{k=1}^{r} \mathcal{B}_{i_{k}}\left(x_{k} ; \lambda_{k}\right) \frac{u_{k}^{i_{k}}}{i_{k} !} \prod_{k=1}^{s} \mathcal{G}_{j_{k}}\left(y_{k} ; \mu_{k}\right) \frac{u_{r+k}^{j_{k}}}{j_{k} !}
\end{aligned}
$$

and $\mathrm{N}_{1}$ and $\mathrm{N}_{2}$ in the right-hand side of (3.20) can be rewritten as

$$
\begin{aligned}
\mathrm{N}_{1}= & n ! \cdot \sum_{I_{r}+J_{s}=n} \sum_{a=1}^{r} \mathcal{P}_{i_{a}}\left(x_{a} ; \lambda\right) \frac{u_{a}}{i_{a} !} \prod_{k=1}^{a-1} \lambda_{k} \mathcal{B}_{i_{k}}\left(x_{k}-x_{a}+1 ; \lambda_{k}\right) \frac{u_{k}^{i_{k}}}{i_{k} !} \\
& \cdot \prod_{k=a+1}^{r} \mathcal{B}_{i_{k}}\left(x_{k}-x_{a} ; \lambda_{k}\right) \frac{u_{k}^{i_{k}}}{i_{k} !} \prod_{k=1}^{s} \mathcal{G}_{j_{k}}\left(y_{k}-x_{a} ; \mu_{k}\right) \frac{u_{r+k}^{j_{k}}}{j_{k} !}
\end{aligned}
$$

and

$$
\begin{aligned}
\mathrm{N}_{2}= & \cdot n ! \cdot \sum_{I_{r}+J_{s}=n} \sum_{b=1}^{s}(-1)^{b} \mathcal{P}_{j_{b}}\left(y_{b} ; \lambda\right) \frac{u_{r+b}}{j_{b} !} \\
& \cdot \prod_{k=1}^{b-1} \mu_{k} \mathcal{G}_{j_{k}}\left(y_{k}-y_{b}+1 ; \mu_{k}\right) \frac{u_{r+k}^{j_{k}}}{j_{k} !} \prod_{k=b+1}^{s} \mathcal{G}_{j_{k}}\left(y_{k}-y_{b} ; \mu_{k}\right) \frac{u_{r+k}^{j_{k}}}{j_{k} !} \\
& \cdot \prod_{k=1}^{r} \lambda_{k} \mathcal{B}_{i_{k}}\left(x_{k}-y_{b}+1 ; \lambda_{k}\right) \frac{u_{k}^{i_{k}}}{i_{k} !} .
\end{aligned}
$$


It follows from (3.20)-(3.23) that

$$
\begin{aligned}
\sum_{I_{r}+J_{s}=n} & \prod_{k=1}^{r} \mathcal{B}_{i_{k}}\left(x_{k} ; \lambda_{k}\right) \frac{u_{k}^{i_{k}}}{i_{k} !} \prod_{k=1}^{s} \mathcal{G}_{j_{k}}\left(y_{k} ; \mu_{k}\right) \frac{u_{r+k}^{j_{k}}}{j_{k} !} \\
= & \sum_{I_{r}+J_{s}=n} \sum_{a=1}^{r} \mathcal{P}_{i_{a}}\left(x_{a} ; \lambda\right) \frac{u_{a}}{i_{a} !} \prod_{k=1}^{a-1} \lambda_{k} \mathcal{B}_{i_{k}}\left(x_{k}-x_{a}+1 ; \lambda_{k}\right) \frac{u_{k}^{i_{k}}}{i_{k} !} \\
& \cdot \prod_{k=a+1}^{r} \mathcal{B}_{i_{k}}\left(x_{k}-x_{a} ; \lambda_{k}\right) \frac{u_{k}^{i_{k}}}{i_{k} !} \prod_{k=1}^{s} \mathcal{G}_{j_{k}}\left(y_{k}-x_{a} ; \mu_{k}\right) \frac{u_{r+k}^{j_{k}}}{j_{k} !} \\
& +2 \sum_{I_{r}+J_{s}=n} \sum_{b=1}^{s}(-1)^{b} \mathcal{P}_{j_{b}}\left(y_{b} ; \lambda\right) \frac{u_{r+b}}{j_{b} !} \\
& \cdot \prod_{k=1}^{b-1} \mu_{k} \mathcal{G}_{j_{k}}\left(y_{k}-y_{b}+1 ; \mu_{k}\right) \frac{u_{r+k}^{j_{k}}}{j_{k} !} \prod_{k=b+1}^{s} \mathcal{G}_{j_{k}}\left(y_{k}-y_{b} ; \mu_{k}\right) \frac{u_{r+k}^{j_{k}}}{j_{k} !} \\
& \cdot \prod_{k=1}^{r} \lambda_{k} \mathcal{B}_{i_{k}}\left(x_{k}-y_{b}+1 ; \lambda_{k}\right) \frac{u_{k}^{i_{k}}}{i_{k} !} .
\end{aligned}
$$

By making the operation $\int \cdots \int_{\Omega_{r+s-1}} \cdot d u_{1} \cdots d u_{r+s-1}$ in both sides of (3.24), with the help of (3.18), we get

$$
\begin{aligned}
& \frac{1}{(n+r+s-1) !} \sum_{I_{r}+s_{s}=n} \prod_{k=1}^{r} \mathcal{B}_{i_{k}}\left(x_{k} ; \lambda_{k}\right) \prod_{k=1}^{s} \mathcal{G}_{j_{k}}\left(y_{k} ; \mu_{k}\right) \\
& =\sum_{I_{r}+J_{s}=n} \sum_{a=1}^{r} \frac{\mathcal{P}_{i_{a}}\left(x_{a} ; \lambda\right)}{i_{a} ! \cdot\left(n-i_{a}+r+s\right) !} \prod_{k=1}^{a-1} \lambda_{k} \mathcal{B}_{i_{k}}\left(x_{k}-x_{a}+1 ; \lambda_{k}\right) \\
& \cdot \prod_{k=a+1}^{r} \mathcal{B}_{i_{k}}\left(x_{k}-x_{a} ; \lambda_{k}\right) \prod_{k=1}^{s} \mathcal{G}_{j_{k}}\left(y_{k}-x_{a} ; \mu_{k}\right) \\
& \quad+2 \sum_{I_{r}+J_{s}=n} \sum_{b=1}^{s}(-1)^{b} \frac{\mathcal{P}_{j_{b}}\left(y_{b} ; \lambda\right)}{j_{b} ! \cdot\left(n-j_{b}+r+s\right) !} \\
& \quad \cdot \prod_{k=1}^{b-1} \mu_{k} \mathcal{G}_{j_{k}}\left(y_{k}-y_{b}+1 ; \mu_{k}\right) \\
& \quad \cdot \prod_{k=b+1}^{s} \mathcal{G}_{j_{k}}\left(y_{k}-y_{b} ; \mu_{k}\right) \prod_{k=1}^{r} \lambda_{k} \mathcal{B}_{i_{k}}\left(x_{k}-y_{b}+1 ; \lambda_{k}\right),
\end{aligned}
$$

as desired. This concludes the proof of Theorem 2 .

Competing interests

The authors declare that they have no competing interests.

\section{Authors' contributions}

All authors participated in drafting, revising, and commenting on the manuscript. All authors read and approved the final manuscript.

\section{Author details}

'Department of Mathematics, Faculty of Science, Kunming University of Science and Technology, Kunming, Yunnan 650500 , People's Republic of China. ${ }^{2}$ Department of Economics, Faculty of Economics, Administrative and Social Sciences, 
Hasan Kalyoncu University, Gaziantep, 27410, Turkey. ${ }^{3}$ Department of Mathematics and Statistics, University of Victoria, Victoria, British Columbia V8W 3R4, Canada. ${ }^{4}$ China Medical University, Taichung, 40402, Taiwan, Republic of China.

\section{Acknowledgements}

We express our sincere thanks to the anonymous referees for their comments on this manuscript. This work was supported by the Foundation for Fostering Talents in Kunming University of Science and Technology (Grant No. KKSY201307047) and the National Natural Science Foundation of the People's Republic of China (Grant No. 11326050).

\section{Received: 27 September 2016 Accepted: 27 October 2016 Published online: 10 November 2016}

\section{References}

1. Agoh, T, Dilcher, K: Convolution identities and lacunary recurrences for Bernoulli numbers. J. Number Theory 124, 105-122 (2007)

2. Araci, S: Novel identities involving Genocchi numbers and polynomials arising from applications of umbral calculus. Appl. Math. Comput. 233, 599-607 (2014)

3. Cohen, H: Number Theory - Volume II: Analytic and Modern Tools. Graduate Texts in Mathematics, vol. 240. Springer, Berlin (2007)

4. Nielsen, N: Traité élémentaire des nombres de Bernoulli. Gauthier-Villars, Paris (1923)

5. Srivastava, HM, Choi, J: Zeta and q-Zeta Functions and Associated Series and Integrals. Elsevier, Amsterdam (2012)

6. Srivastava, HM, Pintér, Á: Remarks on some relationships between the Bernoulli and Euler polynomials. Appl. Math. Lett. 17, 375-380 (2004)

7. Luo, Q-M: Extension for the Genocchi polynomials and its Fourier expansions and integral representations. Osaka J. Math. 48, 291-309 (2011)

8. Luo, Q-M, Srivastava, HM: Some generalizations of the Apostol-Bernoulli and Apostol-Euler polynomials. J. Math. Anal. Appl. 308, 290-302 (2005)

9. Luo, Q-M, Srivastava, HM: Some relationships between the Apostol-Bernoulli and Apostol-Euler polynomials. Comput. Math. Appl. 51, 631-642 (2006)

10. Apostol, TM: On the Lerch zeta function. Pac. J. Math. 1, 161-167 (1951)

11. Srivastava, HM: Some formulas for the Bernoulli and Euler polynomials at rational arguments. Math. Proc. Camb. Philos. Soc. 129, 77-84 (2000)

12. Lu, D-Q, Srivastava, HM: Some series identities involving the generalized Apostol type and related polynomials. Comput. Math. Appl. 62, 3591-3602 (2011)

13. Luo, Q-M: Fourier expansions and integral representations for the Apostol-Bernoulli and Apostol-Euler polynomials. Math. Comput. 78, 2193-2208 (2009)

14. Luo, Q-M, Srivastava, HM: Some generalizations of the Apostol-Genocchi polynomials and the Stirling numbers of the second kind. Appl. Math. Comput. 217, 5702-5728 (2011)

15. Navas, LM, Ruiz, FJ, Varona, JL: Asymptotic estimates for Apostol-Bernoulli and Apostol-Euler polynomials. Math. Comput. 81, 1707-1722 (2011)

16. Prévost, M: Padé approximation and Apostol-Bernoulli and Apostol-Euler polynomials. J. Comput. Appl. Math. 233, 3005-3017 (2010)

17. Srivastava, HM: Some generalizations and basic (or q-) extensions of the Bernoulli, Euler and Genocchi polynomials. Appl. Math. Inf. Sci. 5, 390-444 (2011)

18. Dilcher, K: Sums of products of Bernoulli numbers. J. Number Theory 60, 23-41 (1996)

19. Chen, K-W: Sums of products of generalized Bernoulli polynomials. Pac. J. Math. 208, 39-52 (2003)

20. He, Y, Araci, S: Sums of products of Apostol-Bernoulli and Apostol-Euler polynomials. Adv. Differ. Equ. 2014, Article ID 155 (2014)

21. Huang, I-C, Huang, S-Y: Bernoulli numbers and polynomials via residues. J. Number Theory 76, 178-193 (1999)

22. Kim, M-S, Hu, S: Sums of products of Apostol-Bernoulli numbers. Ramanujan J. 28, 113-123 (2012)

23. Kim, T: Sums of products of $q$-Bernoulli numbers. Arch. Math. 76, 190-195 (2001)

24. Kim, T, Adiga, C: Sums of products of generalized Bernoulli numbers. Int. Math. J. 5, 1-7 (2004)

25. Simsek, Y, Kurt, V, Kim, D: New approach to the complete sum of products of the twisted $(h, q)$-Bernoulli numbers and polynomials. J. Nonlinear Math. Phys. 14, 44-56 (2007)

26. Simsek, Y: Complete sum of products of $(h, a)$-extension of Euler polynomials and numbers. J. Differ. Equ. Appl. 16 $1331-1348$ (2010)

27. Kim, DS, Kim, T, Mansour, T: Euler basis and the product of several Bernoulli and Euler polynomials. Adv. Stud. Contemp. Math. 24, 535-547 (2014)

28. Abramowitz, M, Stegun, IA (eds.): Handbook of Mathematical Functions with Formulas, Graphs, and Mathematical Tables. Applied Mathematics Series, vol. 55. National Bureau of Standards, Washington (1964). Reprinted by Dover, New York, 1965

29. Pan, H, Sun, Z-W: New identities involving Bernoulli and Euler polynomials. J. Comb. Theory, Ser. A 113, 156-175 (2006)

30. Agoh, T: Convolution identities for Bernoulli and Genocchi polynomials. Electron. J. Comb. 21, Article ID P1.65 (2014)

31. Dunne, GV, Schubert, C: Bernoulli number identities from quantum field theory and topological string theory. Commun. Number Theory Phys. 7, 225-249 (2013)

32. Faber, C, Pandharipande, R: Hodge integrals and Gromov-Witten theory. Invent. Math. 139, 173-199 (2000)

33. He, Y, Zhang, WP: Some sum relations involving Bernoulli and Euler polynomials. Integral Transforms Spec. Funct. 22, 207-215 (2011)

34. He, Y, Araci, S, Srivastava, HM, Acikgoz, M: Some new identities for the Apostol-Bernoulli polynomials and the Apostol-Genocchi polynomials. Appl. Math. Comput. 262, 31-41 (2015)

35. Kim, DS, Kim, T, Lee, S-H, Kim, Y-H: Some identities for the product of two Bernoulli and Euler polynomials. Adv. Differ. Equ. 2012, Article ID 95 (2012)

36. Kim, DS, Kim, T: Identities arising from higher-order Daehee polynomial bases. Open Math. 13, 196-208 (2015) 
37. Carlson, BC: Special Functions of Applied Mathematics. Academic Press, New York (1977)

38. Srivastava, HM, Niukkanen, AW: Some Clebsch-Gordan type linearization relations and associated families of Dirichlet integrals. Math. Comput. Model. 37, 245-250 (2003)

39. Andrews, GE: Euler's pentagonal number theorem. Math. Mag. 56, 279-284 (1983)

40. Bell, J: A summary of Euler's work on the pentagonal number theorem. Arch. Hist. Exact Sci. 64, 301-373 (2010)

Submit your manuscript to a SpringerOpen ${ }^{\circ}$ journal and benefit from:

$\rightarrow$ Convenient online submission

Rigorous peer review

- Immediate publication on acceptance

- Open access: articles freely available online

- High visibility within the field

- Retaining the copyright to your article 